\title{
Studies on the Vibration Control Effect of a New Tuned Liquid Damper
}

\author{
Zheng Lu \& Yuling Yang, Jiang Du \\ College of Civil Engineering, Tongji University, Shanghai 200092, China; \\ School of Materials Science and Engineering, Tongji University, Shanghai 200092, China
}

KEYWORD: Karman Vortex; tuned liquid damper; tuned mass damper; passive control; vibration control

ABSTRACT: Tuned liquid dampers (TLD) have been widely applied in the area of vibration control for the advantages of economy, easy implementation and multifunction. However, the traditional TLD needs to be improved because of its narrow control frequency ranges and unsatisfactory capability. Based on a traditional TLD, the scheme of a new TLD is presented in the paper according to the theory of Karman Vortex concept. Related shaking table tests are conducted to study its characteristics and the contrast experiments to a traditional TLD are also conducted in terms of variable mass ratios and frequency ratios. Finally, the results show that the damping effects of the new Vortex based TLD are generally superior to the traditional TLD.

\section{INTRODUCTION}

Tall buildings are designed to have large aspect ratio and use high strength and lightweight materials, the stiffness and damping of the structure decrease continuously, which causes the increased vibration by wind and earthquakes. As a result, additional vibration control devices should be adopted to reduce the vibration, and TLD (Tuned Liquid Damper) is one of the widely used device (Kelly et al. 1972, Yamamoto \& Kawahara 1999). Compared to other devices, TLD has many advantages such as economic, simple, multifunctional, and low maintenance cost (Gunnoo et al. 2016). However, it only has a good vibration damping effect on the structures whose fundamental frequency is close to the tuning frequency.

In this paper, the optimized design based on the theory of Karman Vortex concept has been made and a new TLD is presented. In addition, related shaking table tests are conducted to study its characteristics and the contrast experiments to a traditional TLD are also conducted in terms of variable mass ratios and frequency ratios.

\section{THE PRELIMINARY DESIGN OF THE NEW VORTEX BASED TLD}

Generally, TLD can be divided into shallow water TLD and deep water TLD according to the ratio of the depth of the water in the tank to the length in the direction of vibration. Deep water TLD can be refitted by the life water tank, and has the advantages of low cost and wide-range use, but it also has the disadvantages of lightly modal damping, narrow control frequency ranges and unsatisfactory capability in vibration control. Therefore, the deep water TLD is difficult to be applied in the practice directly.

With same weight, the shallow water TLD is more effective than deep water TLD, while the cost and quantity is high and the area occupation is large. To improve the sloshing damping and the vibration control effect of the deep water TLD, some diaphragms and columns are set in the deep water TLD according to the theory of Karman Vortex. In addition, the purpose of cost reduction and space saving can be achieved. 


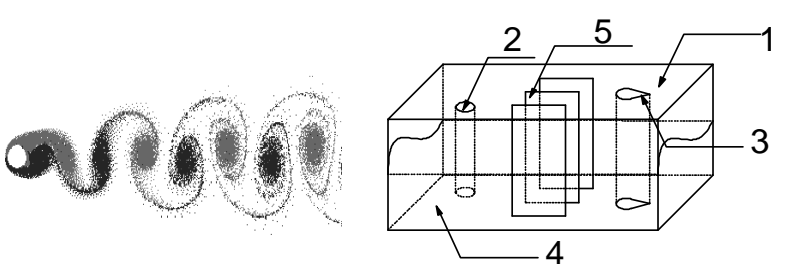

$\begin{array}{ll}\text { (a) Vortex flow around a cylinder } & \text { (b) Scheme of the new TLD }\end{array}$

Fig.1 Concept design of the new vortex based TLD

The Vortex Street is a repeating pattern of alternating swirling vortices caused by the unsteady separation of flow of a fluid around blunt bodies (V. Karman 1912). The diagrammatic sketch of the vortex flow around a cylinder and the scheme of the new TLD is shown in Fig.1. On the basis of a traditional TLD, the cylindrical bluff body, streamline body and rectangular plates are set in the tank. When the water flow through the cylindrical bluff body, the Vortex Street can be formed (SOONG 1997). The streamline body can increase the flow rate and rectangular plates can increase the flow path of water, which will both improve the sloshing damping.

\section{THE DESIGN OF SHAKING TABLE TEST}

\section{Test configuration}

To study the damping effect of the TLD and the factors effecting the vibration reduction performance, related shaking table tests are conducted and the contrast experiments to a traditional TLD are also conducted. The tanks of the new vortex based TLD and traditional TLD are both made of steel, whose height and width are $60 \mathrm{~mm}$, with the length sized $80 \mathrm{~mm}, 100 \mathrm{~mm}, 120 \mathrm{~mm}, 140 \mathrm{~mm}$ and $160 \mathrm{~mm}$ respectively.

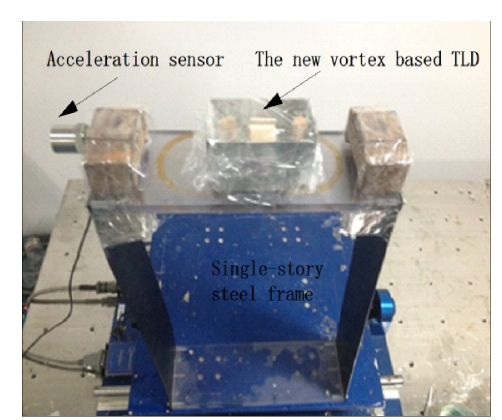

Fig. 2 Test configuration

The test model is a frame structure, with an additional mass weighed $4 \mathrm{~kg}$ on the top, whose frequency is $1.38 \mathrm{~Hz}$, to simulate the ordinary tall buildings. And the acceleration sensor is attached on the top of the structure. The test configuration is shown in Fig.2.

\section{The test parameters}

When the frequency of the traditional deep water TLD is close to the structural fundamental frequency, the damping effect will becomes better with the increase of mass ratio. To study the influence of mass ratios on the performance of the new vortex based TLD, the mass ratios of $2 \%, 3 \%$, $4 \%$ are chosen.

$$
\omega=\sqrt{\frac{\pi g}{L} \tanh \left(\frac{\pi h}{L}\right)}
$$

The natural frequencies and the frequency ratios of the tanks with different mass ratios can be calculated by Formula (1), and the details of TLDs are listed in table 1. The tests about the influence of frequency ratios on the damping effect are also conducted. 
The frequency ratios is defined as the ratio of natural frequency of TLD to the structural natural frequency. And the sine wave, Kobe wave and El-Centro wave are chosen as the structural vibration excitations.

Table 1 Details of TLDs

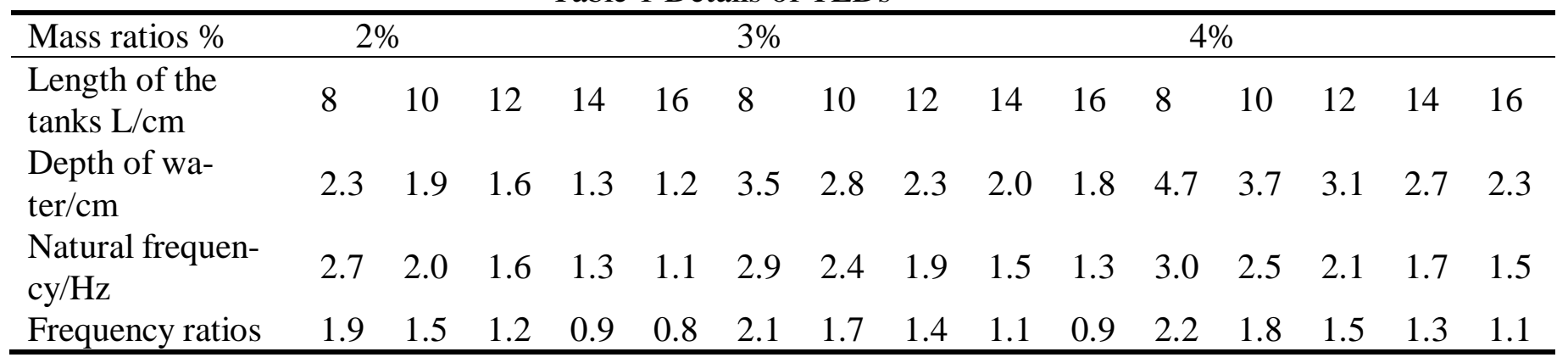

\section{EXPERIMENTAL RESULTS}

\section{The influence of the mass ratios}

To study the influence of the mass ratios, other parameters remain the same, and the frequency ratio is take as 1 .

The vibration reduction effectiveness of the new vortex TLD for different mass ratios is shown in Fig.3. The damping effect becomes better with the increase of the mass ratio under the sine wave. However, the peak and root-mean-square of the acceleration increase at first and then decrease slightly under the Kobe wave and El-Centro wave respectively. This is mainly due to the attachment of the TLD, which increases the mass of the structure and decrease the structural natural frequency. As a result, the natural frequency of TLD diverge from the structural natural frequency, and the damping effect becomes worse.

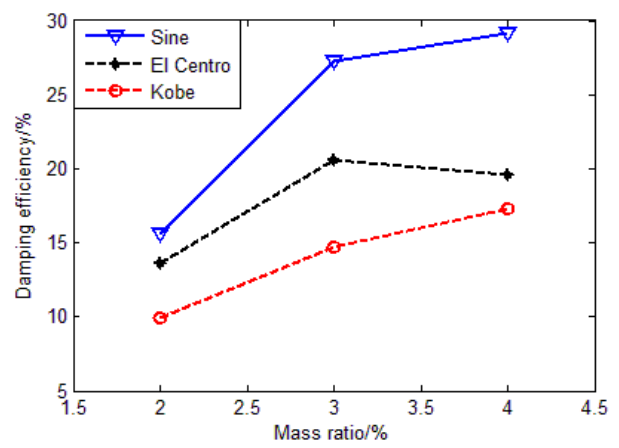

(a) Peak value

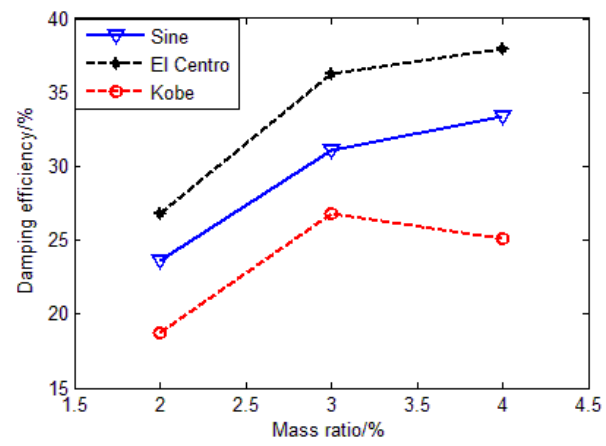

(b) root-mean-square

Fig.3 Vibration reduction effectiveness of the new vortex TLD for different mass ratios 
The acceleration time history curves of the primary structure under sine wave and El Centro wave are shown in Fig4. It is obviously shown that the new vortex based TLD can result in a fast attenuation of the structure response, and has a notable energy-dissipating effect.

In conclusion, the damping effect of the new vortex based TLD becomes better with the increase of the mass ratio, which is consistent with the performance of a traditional TLD. Therefore, the appropriate mass ratio can be chosen, considering the building water consumption demand, to achieve the optimal effect.

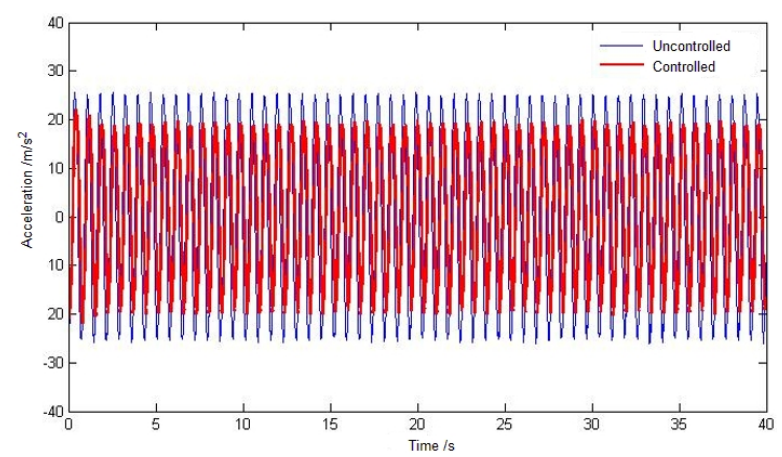

(a) Sine wave

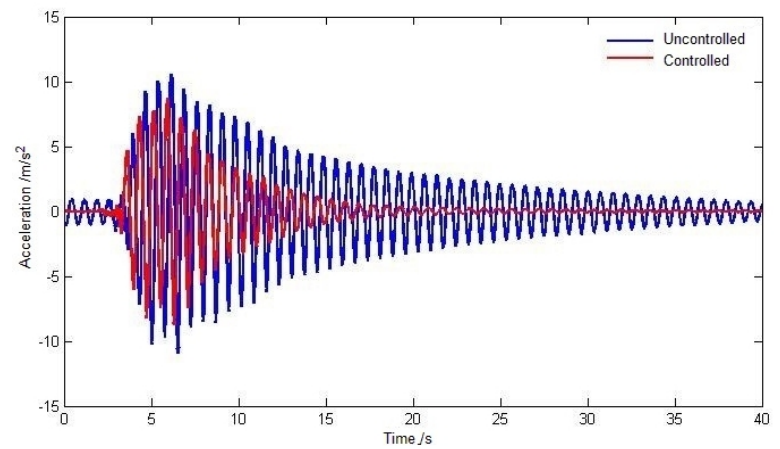

(b) El Centro wave

Fig.4 The acceleration time history curves of the primary structure

\section{The influence of frequency ratios}

It is known from theoretical analysis, the closer the frequency ratio is to 1, the better the damping effect of the traditional TLD is. Considering the internal optimization design of the new TLD, the liquid's flow state and the energy-consuming mechanism are different from the traditional one. The tests about the influence of frequency ratios on the damping effect are also conducted.

The structural natural frequency is determined according to the measured values $\mathrm{f}_{0}=1.38 \mathrm{~Hz}$, and the frequencies of the traditional TLD and the new TLD can be calculated by the Formula (1).

Three series of tests about frequency ratios are conducted under different mass ratios, with $112 \mathrm{~g}$ (the mass ratio of $2 \%$ ), $168 \mathrm{~g}$ (the mass ratio of $3 \%$ ) and $224 \mathrm{~g}$ (the mass ratio of $4 \%$ ) injected into the tank respectively. The details of TLDs are shown in Table.1. To make a contrast of traditional TLD with the new TLD, two tanks in each size are used in the tests. The sine wave, Kobe wave and ElCentro wave are chosen as the structural vibration excitations, with amplitudes being $3 \mathrm{~cm}$.

Taking the damping effect over frequency ratios with the mass ratio of $2 \%$ as an example, the results obtained are analyzed as follows. The damping efficiency of the new vortex TLD for different frequency ratios (with the mass ratio of $2 \%$ ) is shown in Fig.5, and the vibration control superiority of the new vortex TLD compared to the traditional TLD for different frequency ratios is shown in Fig.6. 


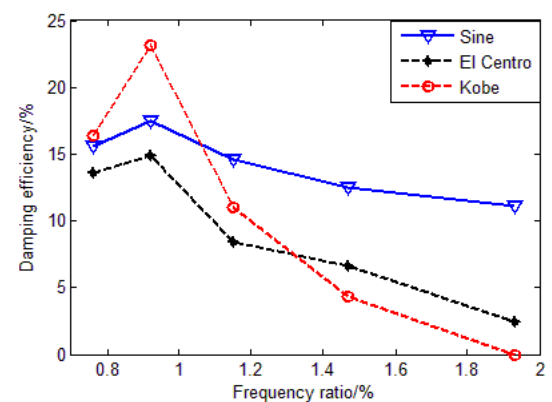

(a) Peak value

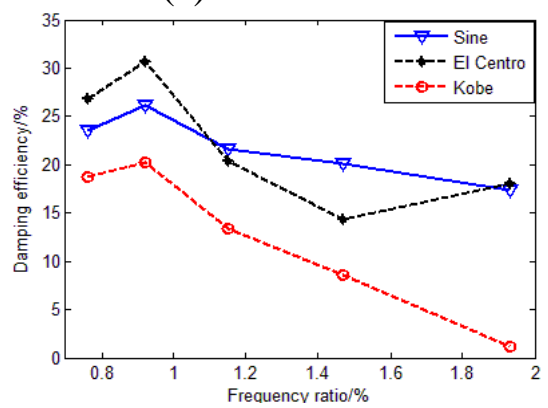

(b) Root-mean-square

Fig.5 Damping efficiency of the new vortex TLD for different frequency ratios (with the mass ratio of $2 \%$ )

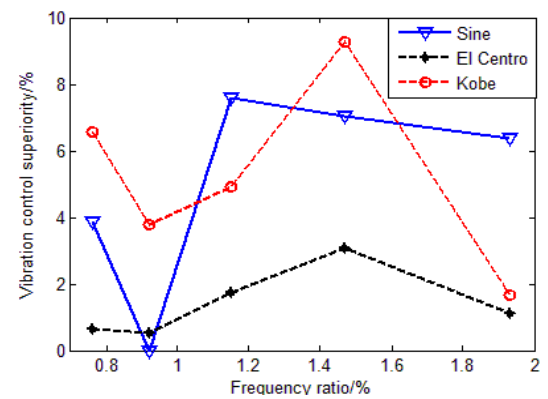

(a) Peak value

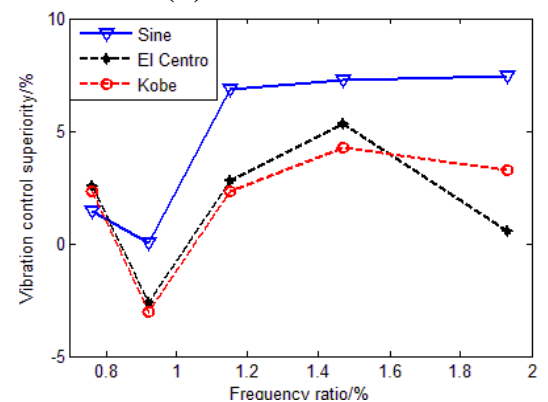

(b) Root-mean-square

Fig.6 Vibration control superiority of the new vortex TLD compared to the traditional TLD for different frequency ratios (with the mass ratio of $2 \%$ )

As seen in Fig.5, the damping efficiency increases gradually over frequency ratios when the frequency ratio is less than 1 . However, when the frequency ratio is larger than 1 , the damping efficiency decrease gradually over frequency ratios, because the natural frequency of TLD diverge from the structural natural frequency and the two are not-tuned.

Fig.6 shows the vibration control superiority of the new vortex TLD. The vibration control superiority is positive in most cases, indicating that the damping effect of the new TLD is generally better than the traditional one. The variation laws of the vibration control superiority is evident. The vibration control superiority decrease over the frequency ratio when the ratio values from 0.7 to 1 , while the superiority increase with the frequency ratio when the ratio values from 1 to 2 . That is, the more 
the frequency of the TLD deviate from the structural natural frequency, the greater the vibration control superiority, which shows that the new vortex based TLD has a wider control frequency ranges compared with the traditional one, and that the new TLD has good robustness in vibration control.

According to the fine analysis of the test results, the damping effect of the new TLD is optimized when the frequency ranges from 0.92 to 1.05 , which is in accordance with the traditional TLD. The new TLD broaden the control frequency ranges significantly, and has better robustness, which has the obvious engineering advantages. On one hand, the damping effect of the dampers will not change rapidly for the depth of the water changes slightly. Thus, special maintenance is not needed, which reduces the cost. On the other hand, the new TLD perform well when the structural natural frequency deviates from the frequency of the TLD for the structural damages under earthquakes.

\section{CONCLUSION}

On the basis of traditional TLD, the scheme of a new TLD is presented in the paper according to the theory of Karman Vortex concept. The cylindrical bluff body, streamline body and rectangular plates are set in the tank to improve the sloshing damping. Related shaking table tests are conducted and the contrast experiments to a traditional TLD are also conducted. Finally, the results show that the damping effects of the new Vortex based TLD are generally superior to the traditional TLD.

The tests shows that damping effect of the new TLD changes over the mass ratios and frequency ratios is in accordance with the traditional TLD. The damping effect of the new TLD, is better than the traditional TLD, with the mass ratio and frequency ratio being equal. In addition, the new TLD has wider control frequency range and better robustness, which has the obvious engineering advantages. Moreover, the new TLD is still effective when the structural natural frequency deviate from the frequency of the TLD for the structural damages under earthquakes, which guarantee good engineering applicability.

\section{ACKNOWLEDGEMENTS}

Financial support from the National Key Technology R\&D Program through grant 2014BAL05B01 is highly appreciated.

\section{REFERENCES}

1) Gunnoo. H, Abcha. N, Ezersky, A. Frequency lock-in and phase synchronization of vortex shedding behind circular cylinder due to surface waves[J]. Physics Letters A, 2016, 380(7-8): 863-868.

2) Kazuya Yamamoto, Mutsuto Kawahara. Structural oscillation control using tuned liquid damper [J]. Computers \& Structures, 1999, 71: 435-446.

3) Kelly J.M., Skinner, R.L., Heine, A.J. Mechanics of energy absorption in special devices for use in earthquake-resistant structures [J]. National Society for Earthquake Engineering, 1972, 5(3): 63-88.

4) SOONG T T, DARGUSH G F. Passive Energy Dissipation System in Structural Engineering [M]. New York: John Wiley\&Sons, 1997: 22-45.

5) V. Karman T. Ueber den Mechanismus des Fluessigkeits und Luftwinderstands [J]. Phys Zeitschrift, 1912, 13: 49-59. 